Robert Langer*

\title{
Bildung für nachhaltige Entwicklung (BNE) als Programm
}

\author{
Das Beispiel der Sächsischen Landesfachstelle für Bibliotheken
}

https://doi.org/10.1515/bfp-2021-0069

Zusammenfassung: Die Sächsische Landesfachstelle für Bibliotheken nimmt sich im 106. Jahr ihres Bestehens des Themas Nachhaltigkeit an. 2020 startete sie ein Kooperationsprojekt zur Einführung von Bildung für nachhaltige Entwicklung (BNE) im öffentlichen Bibliothekswesen. Damit sollen Bibliotheken motiviert werden, das Thema in seinen vielfältigen Facetten wahrzunehmen und zu überlegen, wie und wo es sich in den Arbeitsalltag integrieren lässt.

Schlüsselwörter: Sächsische Landesfachstelle für Bibliotheken; Öffentliche Bibliothek; Bildung für nachhaltige Entwicklung

\section{Saxony's Public Libraries on their way towards BNE The example of the Saxon State Office for Libraries}

Abstract: In its $106^{\text {th }}$ year of existence, the Sächsische Landesfachstelle für Bibliotheken is tackling the issue of sustainability. By starting off a cooperation project to introduce Education for Sustainable Development (ESD) in 2020, the Fachstelle is encouraging public libraries to incorporate the whole range of the topic into their everyday work.

Keywords: Sächsische Landesfachstelle für Bibliotheken; public libraries; Education for Sustainable Development

\section{Einleitung}

In Sachsen werden seit über einhundert Jahren kommunale Bibliotheken durch eine vom Land finanzierte Fachstellenarbeit unterstützt. 1914 in Leipzig als Staatliche Zentralstelle für volkstümliches Büchereiwesen im Königreich Sachsen gegründet, diente sie nicht nur als Bindeglied zwischen den Büchereien und den kommunalen Trägern, sondern unterhielt auch die Fachschule für Bibliotheks-

*Kontaktperson: Dr. Robert Langer, Robert.Langer@lds.sachsen.de technik und -verwaltung. ${ }^{1}$ In der Folge wurden bibliothekarische Beratungsstellen auch in weiteren deutschen Ländern gegründet. Diese staatlichen Beratungsstellen für Öffentliche Büchereien zählen zu den ältesten Einrichtungen des kommunalen Bibliothekswesens. Heute bestehen in den Flächenbundesländern 13 Fachstellen mit unterschiedlicher landeshoheitlicher fachlicher Anbindung, Ausstattung und Aufträgen. Die Bibliotheksfachstellen organisieren sich in der Fachstellenkonferen $z^{2}$ und sind in der Sektion 6 des Deutschen Bibliotheksverbandes (dbv) vertreten. ${ }^{3}$

Die Sächsische Landesfachstelle fördert im gesamten Freistaat die Leistungsfähigkeit der Öffentlichen Bibliotheken mit besonderem Augenmerk auf den ländlichen Raum. Sie gestaltet innovative lokale und regionale Bibliotheksverbünde und vernetzt digitale Angebote der Öffentlichen Bibliotheken untereinander. Sie entwickelt Konzepte zur koordinierten Förderung von Öffentlichen Bibliotheken über die Kulturräume, zur Bibliothekserhaltung und -entwicklung in den Kommunen und zur Zusammenarbeit der Öffentlichen Bibliotheken mit Schulen und anderen Einrichtungen zur Förderung der Lese- und Medienkompetenz. Weiterhin koordiniert die Landesfachstelle die fachbezogene, zielgruppenorientierte Weiterbildung, vermittelt Ergänzungsbestände und bearbeitet die Deutsche Bibliotheksstatistik für den Bereich Öffentliche Bibliotheken des Freistaates Sachsen. Ihr Landesauftrag erstreckt sich auf derzeit 416 Öffentliche Bibliotheken, verteilt auf acht Kulturräume - drei urbane (die Großstädte Dresden, Leipzig und Chemnitz) und fünf ländliche (Oberlausitz-Niederschlesien, Meißen-Sächsische SchweizOsterzgebirge, Erzgebirge-Mittelsachsen, Leipziger Raum und Vogtland-Zwickau).

1 Die heutige Hochschule für Technik, Wirtschaft und Kultur (HTWK) Leipzig ist ihre Nachfolgerin.

2 http://www.fachstellen.de/index.php?p=1 (alle Zugriffe: 29.4.21).

3 https://www.bibliotheksverband.de/fachgruppen/sektionen/sekti on-6.html. 


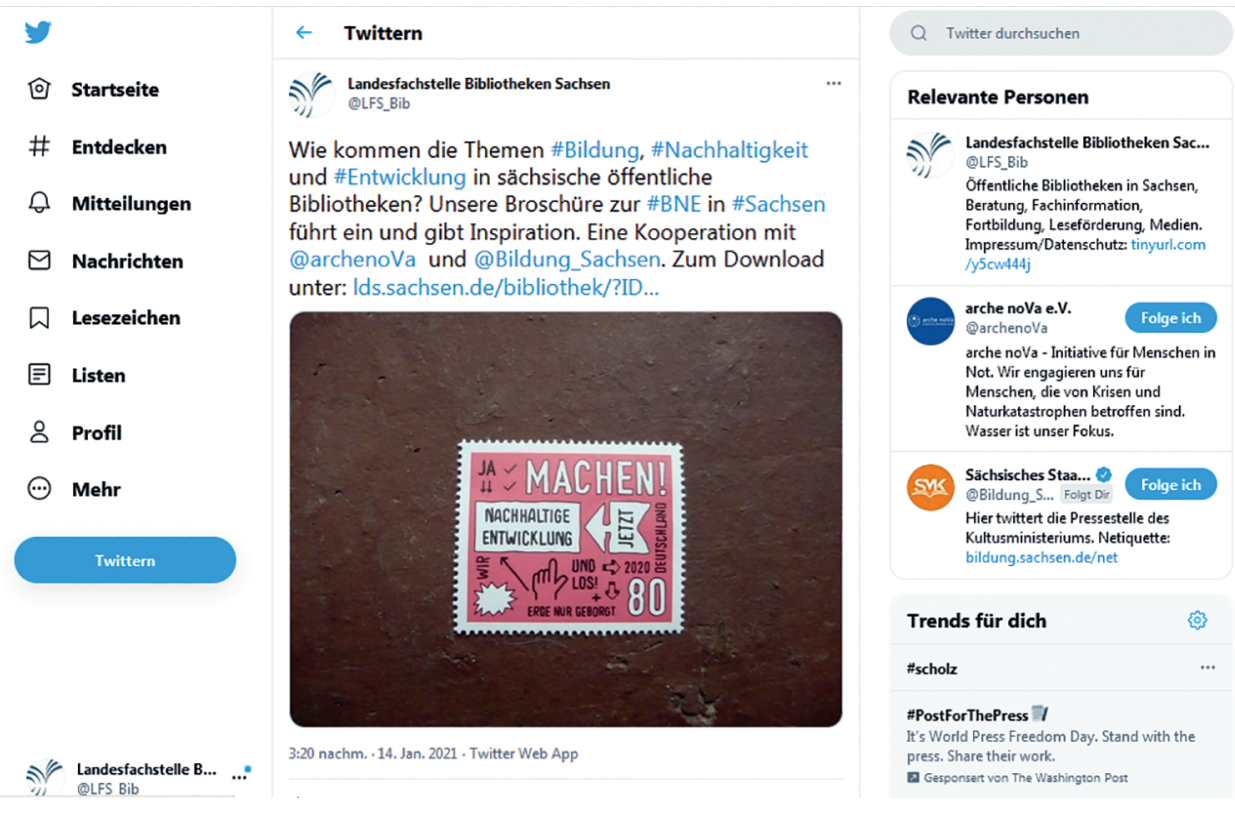

Abb. 1: Tweet der Landesfachstelle zum Thema BNE, (C)ächsische Landesfachstelle für Bibliotheken

\section{BNE in sächsischen Bibliotheken}

Mit der Umsetzung der Landesstrategie Bildung für nachhaltige Entwicklung ${ }^{4}$ ist in Sachsen das Kultusministerium betraut. Dort sieht man Öffentliche Bibliotheken als wünschenswerte Kooperationspartner. Dieses Ansuchen ${ }^{5}$ ist durchaus verständlich, da es dafür kaum Orte gibt, die positiver besetzt sind. 2019 zählten Öffentliche Bibliotheken in Sachsen 6,4 Mio. Besucher, davon entfielen 3 Mio. auf die ländlichen Räume. ${ }^{6}$ Sie zählen damit zu den Magneten, tragen maßgeblich zur Zukunftsfähigkeit der Kommunen bei und leisten einen wichtigen Beitrag zur Erreichung der Nachhaltigkeitsziele der Agenda 2030 der Vereinten Nationen, denn mit ihrer Präsenz in den Städten und Gemeinden haben Bibliotheken eine Vorbildfunktion. Wenn Bibliotheken nachhaltig handeln, kann dies ihre Kommune beeinflussen und die nachhaltige Entwicklung in ihrem Ort vorantreiben. Auch deshalb ist es sinnvoll und wichtig, dass sie unterstützt und gefördert werden.

In diesem Sinne startete die Sächsische Landesfachstelle für Bibliotheken in Kooperation mit dem Verein arche noVa ${ }^{7}$ im September 2020 ein Projekt zur Einführung

\footnotetext{
4 Sachsen. Sächsisches Staatsministerium für Kultus (2018). 5 Deutscher Bibliotheksverband (2021).

6 Alle statistischen Angaben stammen aus der Bibliotheksstatistik des Freistaates Sachsen für das Jahr 2019 als Teil der DBS.

7 https://arche-nova.org/.
}

von Bildung für nachhaltige Entwicklung (BNE) im öffentlichen Bibliothekswesen in Sachsen. ${ }^{8}$

Ausgehend von der Konzeption zur Einführung von BNE in den Leipziger Städtischen Bibliotheken ${ }^{9}$ ging es in einem weiteren Schritt darum, BNE jenseits der Großstädte im öffentlichen Bibliothekswesen zu etablieren. Hierbei sollten Bibliotheken in den Blick genommen werden, die sich in den sächsischen Mittelzentren befinden - sogenannte Mittelpunktbibliotheken. Mittelzentren gelten als regionale Wirtschafts-, Bildungs-, Kultur- und Versorgungszentren, die der Stabilisierung der ländlichen Räume dienen und diese sichern und stärken sollen. Vorgesehen wurde ein zweistufiges Vorgehen. Zuerst sollte es um eine allgemeine Information für alle Öffentlichen Bibliotheken gehen. Es sollten so viele Bibliotheken wie möglich in einer digitalen Weiterbildung für das Thema sensibilisiert werden. Zudem sollten Materialien erstellt werden, die auf der Homepage der Sächsischen Landesfachstelle für Bibliotheken (LFS) bereitgestellt werden und den Bibliotheksbeschäftigten zur Nachnutzung zur Verfügung

8 Arche noVa ist eine in Dresden ansässige humanitäre Organisation, die Menschen in Notsituationen unterstützt, langfristige Entwicklungszusammenarbeit leistet und Bildungsprojekte realisiert. Letztere umfassen neben Grundbildung und Hygieneschulungen in den Projektländern vor allem Globales Lernen und Bildung für Nachhaltige Entwicklung (BNE). Ihre Bildungsarbeit ist zielgruppengerecht und beteiligungsorientiert, legt Wert auf die Aktualität ihrer Materialen, Methoden und Zielsetzungen und orientiert sich an der Alltagswelt der Teilnehmenden.

9 Elsässer und Arnold (2019). 
stehen. Im zweiten Teil sollte je eine Mittelpunktbibliothek aus den fünf ländlichen Kulturräumen bei der Umsetzung der BNE in ihrer Bildungsarbeit beraten werden. Der Anschubprozess sollte begleitet werden, indem mit den Beschäftigten ein Konzept erarbeitet und BNE schließlich implementiert werden sollte.

Bedingt durch die Einschränkungen aufgrund der Coronapandemie konnten individuelle Schulungen und Beratung der Bibliotheken erst ansatzweise beginnen. Hingegen fand eine für alle sächsischen Bibliotheken offene Onlineschulung zum Thema BNE bereits statt. Auch die Handreichung zur „Einführung von Bildung für nachhaltige Entwicklung (BNE) im öffentlichen Bibliothekswesen in Sachsen“10 steht inzwischen als Download auf der Homepage der Landesfachstelle zur Verfügung. Eine kleine Druckauflage nutzt die Fachstelle zur gezielten Öffentlichkeitsarbeit.

\section{Das Konzept zur Integration von BNE in den Bibliotheksalltag}

Inhaltlich beruht die Handreichung auf drei Säulen des Bibliotheksalltags - der nachhaltigen Gestaltung von Arbeitsabläufen und Veranstaltungen sowie der Integration in die Bildungsangebote. Da Veränderung immer bei einem selbst beginnt, werden vor der nach außen gerichteten BNE, Überlegungen zu den hausinternen möglichen Umsetzungen angestellt. Eine Institution, die sich die Frage der Nachhaltigkeit bereits gestellt hat, kann auch offensiv in die Vermittlungsarbeit gehen.

\subsection{Gestaltung von Arbeitsabläufen}

Nachhaltigkeit ist eine Haltung, die sich im Arbeitsalltag widerspiegelt und sich durch alle Arbeitsbereiche zieht. Sie kann auf sehr unterschiedliche Weise erreicht werden. Zum einen geht es um einen ressourcenschonenden Umgang mit allen Arbeitsmaterialien, zum anderen geht es um Arbeitsabläufe, die durch oft nur kleine Veränderungen nachhaltig gestaltet werden können. Die Integration von Nachhaltigkeit in die Arbeitsabläufe der Einrichtung geht von der Analyse des IST-Zustandes über die Entwicklung von Visionen, eine Zielsetzung zur Erarbeitung von Maßnahmen, schließlich deren Umsetzung bis hin zur abschließenden Bewertung. Hier können wiederum drei As-

10 Sächsische Landesfachstelle für Bibliotheken (2020). pekte genauer untersucht werden - Ressourcennutzung, Büroorganisation und Medienbeschaffung. Um sich selbst nicht zu überfordern, scheint es klug zu sein, nach der Machbarkeit der Ideen zu fragen:

- Welche personellen Ressourcen braucht es?

- Welche Maßnahmen implizieren Kosten, welche benötigen keine?

- Von wem braucht die Einrichtung welche Entscheidung?

- Bei welchen Maßnahmen brauchen wir Partner, welche könnten das sein?

- Wo können Ressourcen gespart werden?

- Welche Maßnahmen können wir leicht umsetzen?

In öffentlichen Einrichtungen kann die Beschaffung einen wichtigen Beitrag zur Nachhaltigkeit leisten. Häufig ist die Bibliothek in die Prozesse ihres Trägers eingebunden. Dieser wiederum kann sich für nachhaltige Beschaffung entscheiden, wobei die Bibliothek helfen kann, indem sie Informationen dafür zur Verfügung stellt und entsprechende Vorschläge macht. So soll nachhaltige Beschaffung die Einhaltung von sozialen und ökologischen Mindestanforderungen über die ganze Lieferantenkette einer Organisation auf der Basis ökonomischer Nachhaltigkeit sicherstellen. Ebenso können Umweltaspekte berücksichtigt werden: Muss ein Produkt neu gekauft werden oder stellt die Miete/das Leasing eine umweltfreundlichere Variante dar? Auch kann das Nutzungsende eines Produktes Berücksichtigung finden oder die Frage, wie es um Wiederverwendungsmöglichkeit, Reparierbarkeit und Recyclingfähigkeit bestellt ist.

Auch wenn Öffentliche Bibliotheken nachhaltige Lernorte sind, werden sie jedoch oft in den Gemeinden und Städten nicht als Orte der Nachhaltigkeit gesehen. Viele Bibliotheken kommunizieren nicht nach außen, was sie als Einrichtung in dieser Hinsicht bereits umgesetzt haben. Öffentlichkeitsarbeit ist zeitaufwändig und erfordert personelle Ressourcen, teilweise verfügen Bibliotheken über keine eigene Webseite. Das alles erschwert die Außendarstellung. Hier lohnt es sich, darüber nachzudenken, wie Öffentlichkeitsarbeit mit wenig Aufwand gestaltet werden kann. Denn die Darlegung der eigenen Nachhaltigkeitsstrategie kann als Motivation für das Bibliothekspublikum, aber auch für andere Einrichtungen dienen. Einfach umzusetzende Beispiele hierfür wären Plakate in der Bibliothek mit Informationen darüber, wo welche Ressource gespart wird (,Wir benutzen Papier, das mit dem Blauen Engel zertifiziert ist!“) oder ein Plakat, das darum bittet, das Bibliothekskonto digital zu benutzen (,Wollen Sie den Quittungsausdruck aus Umweltschutzgründen sparen?“). Neuanschaffungen zu Themen der BNE können auf der 
Webseite oder auf einem Büchertisch präsentiert werden. Bereits die Arbeit an einem Nachhaltigkeitskonzept kann dem Publikum mitgeteilt werden. Die IFLA stellt neben anderen Materialien ein Poster - auch in deutscher Sprache - zur Verfügung, das deutlich macht: „Diese Bibliothek unterstützt die Nachhaltigkeitsziele". ${ }^{11}$

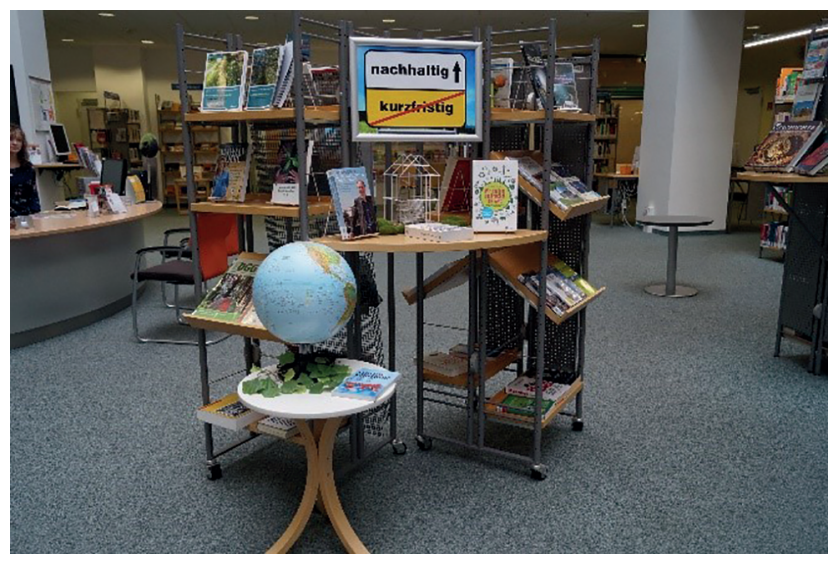

Abb. 2: Informationsstand zur Nachhaltigkeit in der Stadtbibliothek Chemnitz, (OSächsische Landesfachstelle für Bibliotheken

\subsection{Gestaltung von Veranstaltungen}

Nicht jede Veranstaltung, die sich inhaltlich mit Themen der Nachhaltigkeit beschäftigt, ist auch nachhaltig organisiert. Dabei geht es nicht nur um Inhalte, sondern vor allem um die Themen Mobilität, Klima, Energie, Inklusion und Ressourcennutzung. Ein nachhaltiges Veranstaltungsmanagement beginnt bei der Planung der Veranstaltung. Mit allen Beteiligten sollten die nachhaltigen Aspekte im Vorfeld besprochen werden. Hier ist es wichtig abzuwägen, was umsetzbar und wie das Kosten-NutzenVerhältnis ist. Es empfiehlt sich, schon bei der Bewerbung von Veranstaltungen auf Nachhaltigkeitsaspekte hinzuweisen. In die Überlegungen wäre mit einzubeziehen, Veranstaltungsorte in Kooperation mit anderen Einrichtungen in der Kommune zu variieren, um unterschiedliche Zielgruppen $\mathrm{zu}$ erreichen, die Veranstaltungen auf die Fahrpläne der öffentlichen Verkehrsmittel auszurichten oder Fahrpläne in der Bibliothek auszuhängen. Gibt es ausreichend Fahrradstellplätze? Können Flyer besser auf bestimmte Zielgruppen zugeschnitten und kann so die Druckauflage reduziert werden? Im Nachgang kann bei der Bewertung der Veranstaltung durch das Publikum auch der Aspekt der Nachhaltigkeit abgefragt werden. Die

11 https://www.biblio2030.de/materialien/. eigenen Erfahrungen sollte man mit anderen Einrichtungen in der Kommune und anderen Bibliotheken austauschen.

\subsection{Integration von BNE in Bildungsangeboten}

Bildung für nachhaltige Entwicklung richtet sich an alle Zielgruppen. Inklusive Angebote und Angebote für sozial benachteiligte Zielgruppen tragen ebenso zur Umsetzung der Nachhaltigkeitsziele bei, weshalb man sich fragen kann, welche Zielgruppen bisher sehr gut erreicht wurden und warum? Welche Zielgruppen sollen zusätzlich erreicht werden? Wo gibt es Anknüpfungspunkte zur Lebenswelt der Zielgruppe? Wie können Erfahrungen mit neuen Zielgruppen ausgewertet und wie für die weitere Arbeit genutzt werden?

Da BNE die Förderung der Gestaltungskompetenz ${ }^{12}$ und Verhaltensänderungen anstrebt, ist die Nutzung einer breiten Palette von Bildungsformaten besonders wünschenswert. Ungewöhnliche Formate auszuprobieren und mit neuen Formen die Kreativität der Beteiligten und die sinnliche, emotionale Ebene anzusprechen, sollte ebenso in die Angebote der BNE integriert werden wie die rationale Wissensvermittlung.

Um passende Bildungsformate zu entwickeln, kann es von Vorteil sein, sich klarzumachen, welche Formate man anbietet:

- Wie wird welches Format von welcher Zielgruppe aufgenommen?

- Wollen wir neue Formate umsetzen und welche können wir mit den vorhandenen finanziellen, technischen und personellen Kapazitäten umsetzen?

- Mit wem können wir kooperieren, um neue Formate anzubieten?

- Wie werten wir unsere Erfahrungen aus und wie nutzen wir die Erkenntnisse für die weitere Arbeit?

Die Themenpalette zeigt, dass BNE ein Querschnittsthema ist. Nachhaltigkeit betrifft unser gesamtes Leben, ob privat oder im Arbeitskontext. In all unser Tun kann diese Haltung einfließen.

12 https://www.bne-portal.de/de/das-konzept-der-gestaltungs-kom eptenz-2099.html. 


\section{Ausblick}

Die Landesfachstelle hat das Thema BNE nicht nur auf ihrer Homepage $\mathrm{e}^{13}$ verankert, sondern ebenso in ihrem Weiterbildungsprogramm. Zum einen werden die bezüglich BNE geschulten Bibliotheken als Multiplikatoren in ihren Kulturräumen wirken. Auf der anderen Seite knüpfen zukünftige Veranstaltungen an die Erfahrungen an und werden weiter in die ländlichen Räume wirken. Dazu wurden bereits Medienkisten sowohl für Kinder als auch Erwachsene eingerichtet, die bei der Fachstelle für die thematische Bibliotheksarbeit ausgeliehen werden können. ${ }^{14}$

Die bisherigen Schulungen und Beratungen zeigten, dass in sächsischen Öffentlichen Bibliotheken nicht nur ein großes Interesse des Bibliothekspublikums wie auch der Bibliotheksbeschäftigten am Thema BNE besteht, sondern auch vielfältige Potenziale für die Umsetzung und Verankerung von BNE als Querschnittsthema in der täglichen Arbeit der Bibliotheken vorhanden sind. Erste Schritte in Richtung Erweiterung des Medienbestandes um Nachhaltigkeitsthemen, Veranstaltungen zu BNE-Themen, aber auch ressourcenschonende Gestaltung des Bibliotheksbetriebes werden bereits unternommen. Weitere Ideen sind im Entstehen und werden in naher Zukunft wachsen und gedeihen. Als besonderes Zukunftspotenzial wurde die Möglichkeit der stärkeren Vernetzung der Bibliotheken mit anderen BNE Akteuren in Sachsen erkannt. Dabei könnten Synergien hinsichtlich inhaltlicher Expertise, Zielgruppenzugängen, Fördermöglichkeiten und Ressourcen noch besser genutzt werden. So sieht sich die Sächsische Landesfachstelle für Bibliotheken mit ihrem Einsatz zur Implementierung von BNE nicht nur in einer sie prägenden traditionellen Vermittlerrolle, sondern in der Reaktion auf sich wandelnde Entwicklungen als Initiator um die Zukunftsgestaltung des sächsischen Bibliothekswesens.

\section{Literaturverzeichnis}

Deutscher Bibliotheksverband (2021): Die 2030 Agenda für weltweite Entwicklung. Verfügbar unter https://www.bibliotheksverband. de/dbv/themen/agenda-2030.html.

Elsässer, Ralf; Arnold, Antje (2019): Entwicklung einer Konzeption zur Integration von Bildung für nachhaltige Entwicklung (BNE) in kommunalen Bildungseinrichtungen am Beispiel der Leipziger Städtischen Bibliotheken. Verfügbar unter https://static.leip zig.de/fileadmin/mediendatenbank/stadtbibliothek-leipzig-d e/Dokumente/Konzept-BNE-CivixX.pdf.

Sachsen. Sächsisches Staatsministerium für Kultus (2018): Sächsische Landesstrategie Bildung für nachhaltige Entwicklung (BNE). Dresden: Sächsisches Staatsministerium für Kultus. Verfügbar unter https://publikationen.sachsen.de/bdb/artikel/32399/do cuments/49680.

Sächsische Landesfachstelle für Bibliotheken (2020): Bildung für nachhaltige Entwicklung. Verfügbar unter https://www.Ids.sach sen.de/bibliothek/?ID=17185\&art_param=962.

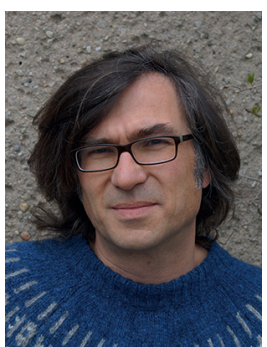

\section{Dr. Robert Langer}

Sächsische Landesfachstelle für Bibliotheken

Zwickauer Straße 56

D-091112 Chemnitz

Robert.Langer@lds.sachsen.de

$13 \mathrm{https://www.lds.sachsen.de/bibliothek/?ID=4215 \& art \_ param=3}$

79.

14 https://www.lds.sachsen.de/bibliothek/?ID=17546\&art_param=9

62. 